\title{
Techniques of uniportal video-assisted thoracic surgery-esophageal and mediastinal indications
}

\author{
Hasan F. Batirel \\ Thoracic Surgery Department, Marmara University Hospital, Istanbul, Turkey \\ Correspondence to: Hasan F. Batirel, MD, PhD. Thoracic Surgery Department, Marmara University Hospital, 7. Kat, Gogus Cerrahisi. Fevzi Cakmak \\ Mah., Mimar Sinan Cad., No: 41, 34899, Ust Kaynarca, Pendik, Istanbul, Turkey. Email: hbatirel@marmara.edu.tr.
}

\begin{abstract}
Uniportal approach for esophagus and mediastinum is gaining popularity. While a transthoracic approach is applied for esophagus frequently from the 5th or 6th intercostal space on the posterior axillary line, approach to anterior mediastinum is variable with access through right/left chest, cervical and subxiphoid regions. The results of uniportal approach for esophagus and mediastinum are comparable with multiport video-assisted thoracic surgery (VATS) and open approach in terms of bleeding, oncologic adequacy and operation times. Indications are similar with open and multiportal VATS cases, however large mediastinal tumors $(>5 \mathrm{~cm}$ ) and T3-4 esophageal cancers can be challenging in the beginning in terms of oncologic adequacy of the operations. Uniportal approach for esophagus and mediastinum is utilized more frequently and initial reports show that it is feasible and its applicability and advantages will become apparent in the coming years.
\end{abstract}

Keywords: Esophagus; mediastinum; uniportal VATS; thymoma; esophageal cancer

Submitted Jun 24, 2019. Accepted for publication Sep 18, 2019.

doi: $10.21037 /$ jtd.2019.09.55

View this article at: http://dx.doi.org/10.21037/jtd.2019.09.55

Techniques of video-assisted thoracic surgery (VATS) evolved from multiportal approach to biportal and uniportal approaches over the last decade (1-3). The change has been fast and adoption of uniportal technique is increasing rapidly. However, uniportal approach to esophageal and mediastinal pathologies are rare and multiportal VATS for esophageal and mediastinal pathologies is still the most commonly applied approach (4).

Classical VATS approach for esophageal cancer and mediastinal tumors still uses at least 3-4 incisions to allow facilitated intrathoracic movements, angles for instruments and suturing (5). There are challenges in both anatomic locations as these structures are located in anterior and posterior mediastinum with narrow spaces. Thus uniportal technique for these locations needs a special technical planning. We have previously demonstrated that uniportal esophageal mobilization or esophageal resection/ anastomosis is feasible when VATS technique is refined and correctly applied (4). In this article we describe indications and technical details of uniportal technique for esophagus and mediastinum.

\section{Esophagus}

\section{Positioning and ıncision}

Esophagus is in the posterior mediastinum, thus the location of the incision should differ from a routine uniportal incision for lung resection.

It is comfortable to use left lateral decubitus position for the patient. The patient is tilted 30 degrees anteriorly which flips the lung to anterior mediastinum. Patient can also be in a full lateral decubitus position and the operating table can be tilted anteriorly during surgery. The patient needs to be secured and draped accordingly.

We prefer a 5 th or 6th intercostal space incision on the posterior axillary line based on patient's physical characteristics (Figure 1). We prefer a 6th intercostal space if the chest cavity is long and shallow and a 5 th intercostal space access if the patient is obese with a short and wide 


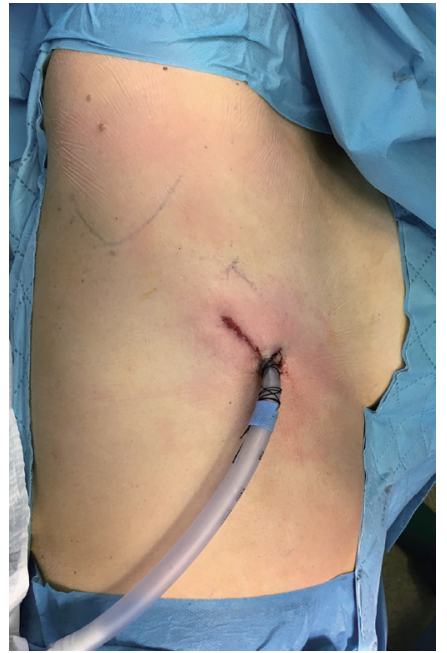

Figure 1 The uniportal incision is made on the 5 th or 6 th intercostal space, posterior axillary line. In this patient with uniportal esophageal mobilization the incision was placed on the 6th intercostal space. If the patient is obese with a short chest we can place our incision on the 5 th intercostal space, whereas if the patient is thin with shallow and long chest, incision can be placed on the 6 th intercostal space.

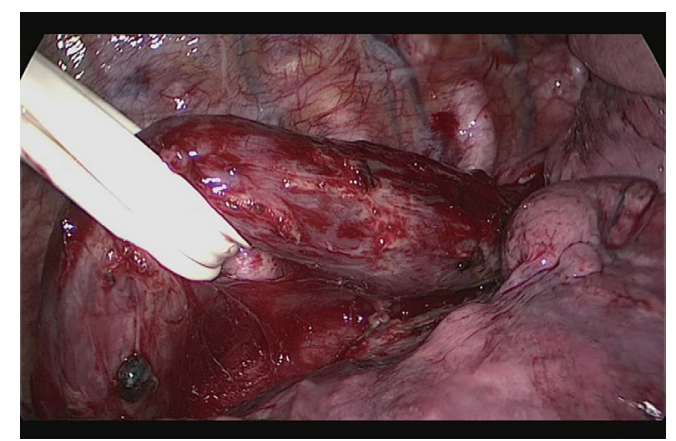

Figure 2 Esophagus is encircled with a $2 \mathrm{~cm}$ penrose drain. Penrose drain can be retracted to various locations facilitating visualization of mediastinal structures.

chest cavity with a sizable liver. Adjusting the incision accordingly allows easier dissection and better positioning of the chest tube. Long thoracoscopic instruments are preferred and especially helpful during dissection of the esophagus at the apex.

A recently published case report utilized a lower posterior incision close to the mid-scapular line to have direct access to the posterior mediastinum (6). Obviously at this location, intercostal space is narrow, leading to crowding of instruments, difficulty to pass the staplers and thus increasing the likelihood of nerve injury.

\section{Peculiarities of dissection/resection}

Chest cavity is inspected for adhesions and in case of a cancer, pleural implants, invasion of mediastinal structures or lung metastasis should be assessed.

During esophageal mobilization for esophageal cancer, the lower lobe is retracted anterosuperiorly. Inferior pulmonary ligament is divided and station 9 lymph nodes are mobilized en bloc with the esophagus. If the tumor is located in lower or middle third, and the patient had neoadjuvant chemoradiation, it is important to identify the pericardium in the medial part of inferior pulmonary vein and use it as a guide for dissection.

Mediastinal pleura is incised adjacent to the lung parenchyma over the inferior pulmonary vein and intermediary bronchus to the level of the azygos vein anteriorly and until the hiatus anterior to the hemiazygos vein posteriorly. Azygos vein is divided with an endoscopic vascular stapler.

Subcarinal lymph node is dissected off the pericardium and intermediary bronchus until the carina. Dissection can be advanced posterior to the carina and to the membranous part of the trachea. Posterior to the pericardium, the plane is relatively avascular and can be divided until contralateral pulmonary veins are visualized. The fatty tissue between hemiazygos vein and esophagus is divided and descending aorta is visualized. Small aortic branches are divided with energy devices and dissection is carried out until we see the left vagus nerve and contralateral pleura/lung. A right angle clamp is passed from anterior to posterior, esophagus is lifted and encircled with a $2 \mathrm{~cm}$ thick penrose drain (Figure 2). When the penrose drain is retracted anteriorly at the bottom of the incision, there is no further need for lung retraction. An angled forceps with a peanut can help lift the esophagus to the anterior side opening the plane between left main bronchus and esophagus. During dissection of the subcarinal lymph node which extends medial to the left main bronchus, the penrose drain is grasped very close to the esophagus with a ring forceps and the ring forceps is pushed inside the chest at the apex of the incision, lifting the esophagus laterally and posteriorly. This maneuver shows the anatomic plane between carina, left main bronchus and the subcarinal lymph node (Figure 3). The lymph node is then freed and esophagus is mobilized.

Above the carina, the dissection is carried out close to the 


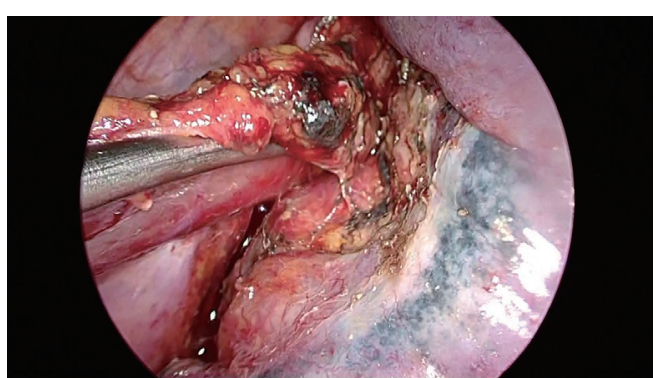

Figure 3 When penrose drain is retracted laterally and posteriorly, left main bronchus and retrotracheal area is clearly visualized.

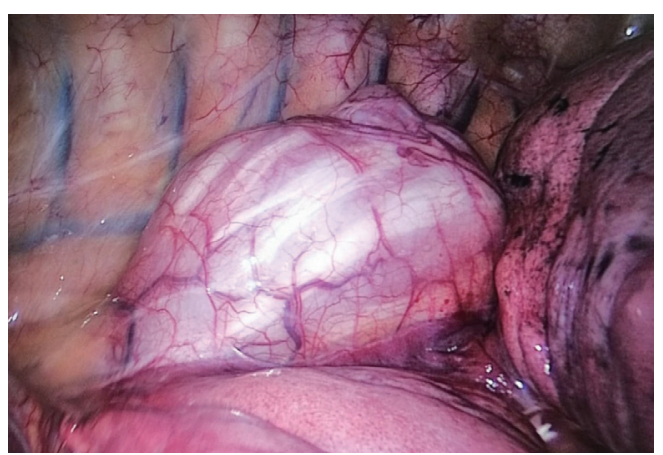

Figure 4 Visualization of a large esophageal leiomyoma in a 35-year-old male from a uniportal VATS approach. VATS, videoassisted thoracic surgery.

esophagus and vagal nerves are divided. Inferiorly all the tissues between inferior vena cava and descending aorta are dissected and mediastinal pleura and attachments close to the diaphragmatic crus are freed. All periesophageal lymph nodes are resected en bloc with the specimen. At this stage all intrathoracic esophagus is freed and visualized.

If the anastomosis is to be performed in the cervical region, the penrose drain is tied around the esophagus and pushed posteriorly to the neck for later retrieval, facilitating identification of esophagus and preventing injury to the recurrent laryngeal nerves.

A single chest tube (preferably 32 Fr) is placed from the anterior part of the incision, aligned inferiorly and advancing to the apex from the paravertebral sulcus.

In case of benign diseases, such as esophageal motility problems, leiomyomas or diverticulas, the same incision is used (Figure 4). Longitudinal and/or circular muscle of the esophagus is cut using energy devices and tumor is excised or myotomy is performed. In case of an intrathoracic diverticula, adhesions of the diverticula should be freed.
Once the neck of the diverticula is identified, the neck is divided with stapler. Muscle layer over the diverticula is closed and a contralateral myotomy is performed.

\section{Reconstruction/anastomosis}

In case of a minimally invasive Ivor Lewis esophagectomy (MI-ILE), an intrathoracic anastomosis between gastric conduit and esophagus is required. The mobilization technique of the esophagus is the same. We avoid very high dissection of the esophagus at the apex of the chest. Once intrathoracic esophagus is mobilized, gastroesophageal block is pulled to the chest in the same orientation. The stapler line should be facing the lateral wall of the chest.

Following this, the stomach conduit is divided with a $60 \mathrm{~mm}$ thick tissue endoscopic stapler at its tip. Length of the conduit is checked before division of the esophagus in order to avoid anastomotic tension. Usually 16-20 cm of gastric conduit reaches the apex of the chest. Then the esophagus is divided $1-2 \mathrm{~cm}$ above the azygos vein with a $60 \mathrm{~mm}$ endoscopic stapler. Stapler is placed from the apex of the incision and angle is applied if necessary. It is important to make sure that nasogastric tube is completely removed at this stage.

Anastomotic technique is a side to side completely stapled anastomosis. No. 1 silk suture is placed at the stapler line of the esophagus. Esophageal stump just under the stapler line is cut and mucosa is visualized. A nasogastric tube is pushed out of the opening. Then stomach conduit is pulled out of the port incision in correct orientation. A 6-7 mm gastrotomy is made $3-4 \mathrm{~cm}$ distal to the tip of the stomach conduit. Gastrostomy can be placed anterior or posteriorly. Another no 1 silk suture is placed at the tip of the stomach conduit for retraction. The thicker leg of a $60 \mathrm{~mm}$ thick tissue endoscopic stapler is placed inside the stomach. The stapler is advanced inside the chest while applying gentle traction to both silk sutures. Camera is at the apex and stapler is in the middle of the incision. No angle is applied to the stapler. The thin leg of the stapler is advanced inside the esophagus taking the nasogastric tube as a guide. Once both legs are inside the esophagus and stomach, nasogastric tube is completely removed. Edges of esophagus and stomach are aligned equally and stapler is fired to form the posterior side of the anastomosis (Figure 5). Both silk sutures are lifted inside the chest towards the lateral chest wall. Usually two firings of $60 \mathrm{~mm}$ endoscopic stapler completes the side esophagogastrostomy (Figure 6). The stapler is fully angled and placed from the apex of the 


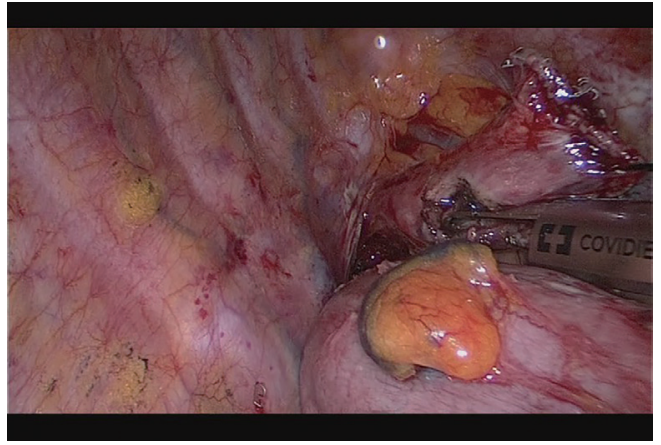

Figure 5 A $60 \mathrm{~mm}$ endoscopic tissue stapler is inserted in the gastric conduit and the esophagus to form the posterior wall of the anastomosis.

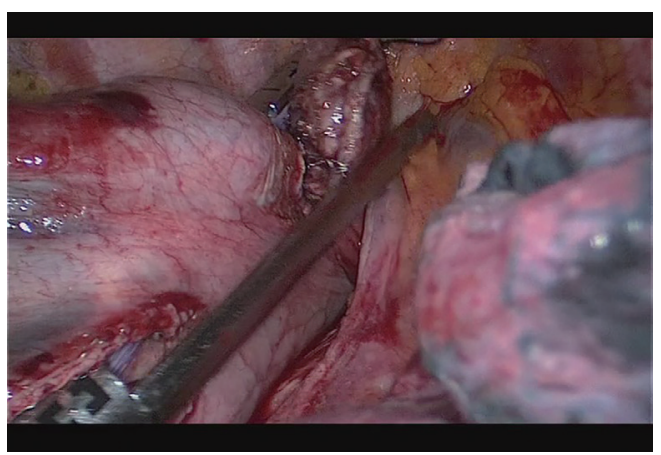

Figure 6 The lateral wall of the stomach and esophagus is divided with an endoscopic thick tissue stapler which completes the anastomosis.

incision. A nasogastric tube can be advanced at this stage of the operation. Stapler line is checked for its contact with membranous part of the trachea and can be covered with omentum if necessary. If a nasogastric tube is placed, air is insufflated and stapler line is checked for leak and integrity.

\section{Postoperative management}

The patient can be taken to the ward or intensive care unit, depending on patient factors. For patients with no known risk factors and younger than 70 , we take them to the ward and start oral feeding on day 3 if gastrointestinal motility is evident and there is no clinical sign of leak. Routine postoperative follow-up is performed with chest X-rays, blood counts and laboratory investigation.

Oral feeding is slowly advanced depending on gastric emptying. If postoperative course is uneventful, the patient is usually discharged on day 7 .

\section{Mediastinum}

There are several access sites to the mediastinum. Most of the procedures involve anterior mediastinum and in literature cervical, transthoracic and subxiphoid approaches have all been described (7-9). In the middle or posterior mediastinum a uniportal approach is also possible and commonly bronchogenic cysts, pericardial cysts and neurogenic tumors can be removed through this incision.

\section{Positioning and incision}

For middle and posterior mediastinum, the position is very similar to esophageal approach and should be a lateral decubitus position with anterior tilt. Incision should be placed on the 5 th or 6 th intercostal space, posterior axillary line, depending on the location of the lesion and patient characteristics.

For anterior mediastinum the patient is positioned supine with a pillow under the scapula placed horizontally. Three types of incisions can be performed for removal of anterior mediastinal lesions:

* A 3-5 cm transverse Collar incision is performed in the neck and typically sternal elevator is placed to lift the sternum and widen the anterior mediastinal space;

* A 3-7 cm longitudinal subxiphoid incision is performed depending on the size of the lesion and etiology and sternal elevator is placed to lift the sternum;

- Right or left transthoracic uniportal approach on the 4th or 5th intercostal space anterior axillary line depending on the location and size of the lesion (Figure 7). A higher intercostal space is preferred on the left side to avoid the heart.

\section{Peculiarities of dissection/resection}

The types of instruments differ depending on the approach, however an energy device for dissection and division, a curved suction and standard minimally invasive surgery instruments are enough. For subxiphoid approach carbon dioxide insufflation is also used to facilitate dissection and obviate anatomic planes. Double lumen intubation is preferred in uniportal VATS approach to deflate the lung and allow for better visualization. 


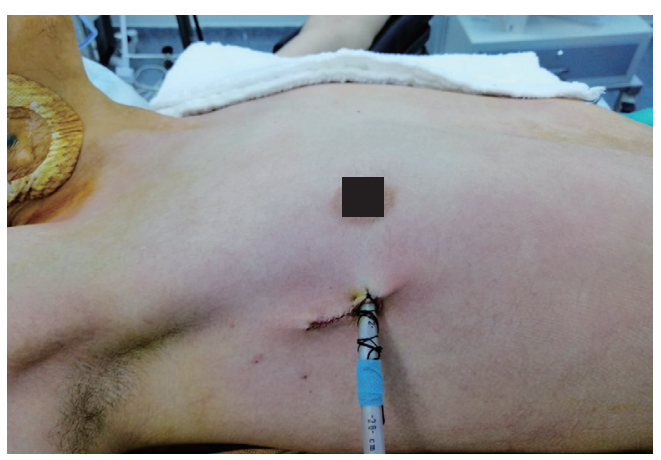

Figure 7 Right transthoracic uniportal approach for a thymoma is seen from the 4 th or 5 th intercostal space anterior axillary line.

Neurogenic tumors are dissected through a plane formed by cutting the parietal pleura and lifting up the tumor. It is crucial to make sure that there is no intraforaminal extension. These tumors usually originate from the sympathetic chain and involved ganglia should be removed en bloc with the tumor as well.

Bronchogenic cysts are typically located in the paratracheal or subcarinal area. There is usually significant peribronchial inflammation and enlarged bronchial and collateral vessels should be sealed using energy devices. Complete removal is essential and sometimes thick adhesions exist between the cyst, subcarinal lymph node and main bronchi-carina.

Transcervical approach is well known and had been used for a long time for removal of thymus in myasthenia gravis (9). The first step is to free the gland from both sides, identifying the brachiocephalic vein and dividing the thymic veins. Once the thymus is free it can be removed and extended resection of fatty tissue can be carried out until the diaphragm and both phrenic nerves.

For subxiphoid anterior mediastinal approach, it is recommended to open both pleura from the beginning to flip the specimen from one side to another and allow visualization of small vessels and dissection planes (8). This maneuver also allows visualization and clipping of thymic veins and vessels. Both phrenic nerves can be clearly visualized from the subxiphoid approach allowing safe dissection.

For VATS approach, mediastinal fatty tissue should be freed from the ipsilateral nerve and the diaphragm extending the dissection towards the brachiocephalic vein. Superior vena cava and drainage site of the internal mammary vein on the right side is an anatomical guide to open a plane over the brachiocephalic vein and identifying thymic vessels. On the left side brachiocephalic vein can be identified higher in the mediastinum and dissection can be advanced towards the right side.

\section{Postoperative management}

Uniportal mediastinal surgery is commonly a surgery without complications and a single drain is placed through the same incision in case of subxiphoid and VATS approaches. A small silicon drain can be placed from the neck incision in case of a transcervical approach. The patient is extubated in the operation room and taken to the ward. Early mobilization and oral feeding is encouraged. Non-steroidal anti-inflammatory drugs are usually sufficient for pain. The tube is removed the next or second day after surgery depending on the amount $(<200 \mathrm{~mL})$ of the drainage. The patient can be discharged on day 3 or 4 .

\section{Discussion}

Uniportal approach for esophagus and mediastinum is developing and will become popularized. However the progress is slower than the lung, as the pathologies are rarer and there is a need for optimization of the technique. All minimally invasive techniques should be comparable to open technique and each other in terms of duration of surgery, amount of bleeding, lymph node yield and leak rates. Additionally the technique should be reproducible in order to be adapted worldwide. The use of less number of incisions would be expected to decrease trauma to the patient.

In our approach for uniportal esophageal surgery, the technique has certain maneuvers that simplify the procedure. One of them is no handling or grasping of the regions of the esophagus and stomach where the anastomosis will be performed. Second is a completely side to side stapled anastomotic technique which is simple and easy to perform. Our abstract on 18 patients who underwent uniportal VATS esophagectomy (16 cases with Ivor Lewis) for esophageal cancer showed that there was no perioperative mortality (10). Average number of lymph nodes was $23 \pm 8$ and mean duration of VATS was $82 \pm 22$ minutes. Eight patients had preoperative chemoradiation. Three patients had leaks. Two occurred in the chest (1 late 22 days after surgery), 1 in the neck.

Uniportal esophageal approach was also reported by Nachira and colleagues in 2018 in 12 patients (11). Two patients had early leak. Mean operative VATS time was 
$105 \pm 21$ minutes. Average number of lymph nodes removed was $10.4 \pm 4$. All patients had neck anastomosis. Mean and median hospital stays were $16 \pm 14$ and 9 respectively. There was another single patient report by Caronia in 2018 (6). The incision was posteriorly located and a circular stapled anastomosis was performed in the chest.

Intrathoracic esophagogastric anastomosis is a rate limiting step for popularization of minimally invasive Ivor Lewis esophagectomy. Experience and optimization of the technique lead to very low rate of leak or even no leak. A recent report showed that there were no leaks after 130 cases until 170 (12).

For uniportal mediastinal surgery, the main concern is in case of thymomas. Thymomas are associated with excellent long term survival if they are completely resected without disruption of the capsule of the tumor. Thus careful surgical technique is a must in thymomas and in case of any suspicion of oncologic adequacy, an open approach should be adopted. Tumors larger than $4-5 \mathrm{~cm}$ and with suspected vascular invasion should be excluded from a minimally invasive approach (13). A no touch technique should be used during handling of a thymic tumor. In case of a thymectomy for myasthenia gravis, complete removal of mediastinal fat should be the aim and this is possible with uni- or multiportal VATS technique. For subxiphoid approach, removal of the xiphoid is recommended if it is ossified. It is recommended to perform a slightly lower incision on the midline and suturing of the rectus muscles to prevent postoperative hernia. Zieliński reported 14 patients with thymomas and 75 patients for non thymomatous myasthenia gravis using a subxiphoid approach (8). The diameter of the thymomas ranged from $3 \mathrm{~cm} \times 3 \mathrm{~cm} \times 2.5 \mathrm{~cm}$ to $12 \mathrm{~cm} \times 9 \mathrm{~cm} \times 5 \mathrm{~cm}$. The mean operative time was $120 \mathrm{~min}(75-155 \mathrm{~min})$ with no perioperative morbidity and only one morbidity. The limitations of a subxiphoid approach were stated as difficulty of manipulation in large thymomas and poor visualization of the recurrent laryngeal nerves and fatty tissues in the neck.

Another paper from Taiwan analyzed 40 patients who underwent mediastinal surgery using a uniportal approach through a 4-5th intercostal space incision on the anterior axillary line (7). Outcomes were excellent with a mean operation time of $97 \pm 31$ minutes and no mortality or morbidity. Average hospital stay was $3.7 \pm 1.6$ days.

In conclusion, uniportal approach for esophagus and mediastinum can be adopted and initial series with significant number of patients show that it is feasible. Currently the technique leads to excellent short term outcomes, however long term results such as oncologic adequacy and herniation will determine the adoption of the technique in the global thoracic surgical community.

\section{Acknowledgments}

None.

\section{Footnote}

Conflicts of Interest: Dr. Batirel is a consultant with Johnson and Johnson and receives fees and honoraria.

Ethical Statement: The author is accountable for all aspects of the work in ensuring that questions related to the accuracy or integrity of any part of the work are appropriately investigated and resolved. Written informed consent was obtained from the patient for publication of this manuscript and any accompanying images.

\section{References}

1. Luketich JD, Alvelo-Rivera M, Buenaventura PO, et al. Minimally invasive esophagectomy: outcomes in 222 patients. Ann Surg 2003;238:486-94; discussion 494-5.

2. Zhang J, Wang R, Liu S, et al. Refinement of minimally invasive esophagectomy techniques after 15 years of experience. J Gastrointest Surg 2012;16:1768-74.

3. Luketich JD, Pennathur A, Awais O, et al. Outcomes after minimally invasive esophagectomy: review of over 1000 patients. Ann Surg 2012;256:95-103.

4. Batirel HF. Uniportal video-assisted thoracic surgery for esophageal cancer. J Vis Surg 2017;3:156.

5. Pennathur A, Awais O, Luketich JD. Technique of minimally invasive Ivor Lewis esophagectomy. Ann Thorac Surg 2010;89:S2159-62.

6. Caronia FP, Arrigo E, Failla AV, et al. Uniportal thoracoscopy combined with laparoscopy as minimally invasive treatment of esophageal cancer. J Thorac Dis 2018;10:E265-9.

7. Wu CY, Heish MJ, Wu CF. Single port VATS mediastinal tumor resection: Taiwan experience. Ann Cardiothorac Surg 2016;5:107-11.

8. Zieliński M, Rybak M, Wilkojc M, et al. Subxiphoid video-assisted thorascopic thymectomy for thymoma. Ann Cardiothorac Surg 2015;4:564-6.

9. Migliore M, Criscione A, Nardini M, et al. Single incision extended video assisted transcervical thymectomy. J Vis 
Surg 2017;3:154.

10. Batirel HF, Durusoy AF, Cetinkaya C, et al. Uniportal thoracoscopic approach for esophageal cancer: Feasibility and Outcome. 27th European Society of Thoracic Surgeons Meeting, Abstract no P-208, Dublin, Ireland, 9-12 June 2019.

11. Nachira D, Meacci E, Mastromarino MG, et al. Initial experience with uniportal video-assisted thoracic surgery

Cite this article as: Batirel HF. Techniques of uniportal video-assisted thoracic surgery-esophageal and mediastinal indications. J Thorac Dis 2019;11(Suppl 16):S2108-S2114. doi: $10.21037 /$ jtd.2019.09.55 esophagectomy. J Thorac Dis 2018;10:S3686-95.

12. White A, Kucukak S, Lee DN, et al. Ivor Lewis minimally invasive esophagectomy for esophageal cancer: An excellent operation that improves with experience. J Thorac Cardiovasc Surg 2019;157:783-9.

13. Bedetti B, Solli P, Lawrence D, et al. Single port videoassisted thoracoscopic thymectomy. J Vis Surg 2016;2:149. 